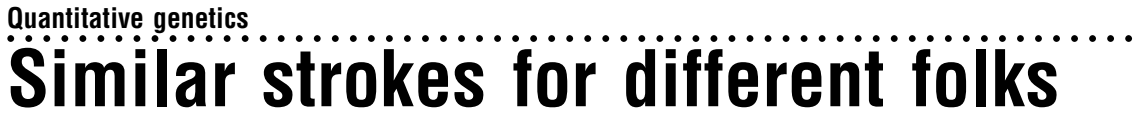
KE Holsinger

Heredity (2006) 96,203 doi:101038/sj hdy 6800770 ; published online 9 November 2005

n January, 2005 Lawrence Summers, President of Harvard University, ignited a firestorm when he suggested that the small number of women on science faculties reflects intrinsic differences in the distribution of scientific aptitude between men and women. While he may have been wrong about those differences, men and women are different in many ways that are unrelated to their roles in reproduction, just as males and females of many animals differ in body size, coloration, and a host of other features.

In many species, differences between the sexes have been explained as a consequence of differences in the natural selection experienced by males and females. However, explaining differences requires not just understanding the selection, but also the genetic response to that selection. In this month's Heredity there is a striking example of how challenging understanding genetic responses can be for sex-specific differences. Parker and Garant (2005) show that male and female red jungle fowl (Gallus gallus) have evolved striking differences in their respective growth trajectories in spite of very high genetic correlations between both the shape and timing of those trajectories.

The functional significance of differences in body size is clear. In many animals, larger males compete more effectively for mates than smaller ones, and they leave more offspring. Bateman's principle (1948) tells us that differences in reproductive success tend to be much larger among males than among females. One consequence of this principle is that selection favors large body size more strongly in males than in females. If large males tend to have large offspring, males will evolve towards larger body sizes than females. Thus, behavioral ecologists typically conclude that males and females differ in body size because of selection associated with male-male competition for females. For example, both body mass and tarsus length are positively correlated with dominance rank and repro- ductive success in male jungle fowl, probably because of associated advantages in male-male competition (Parker et al, 2002). In this new study, the authors show that both tarsus length and body mass are heritable at almost every age where it was assessed. As might be expected, from this understanding of selection, male jungle fowl are also much larger than females (Parker and Garant, 2005).

However, the genetic response to selection is not so straightforward: males and females share the bulk of their genes, so we would expect the sexes to share many genes that influence body size. If they do, we might also expect body size in males and females to be genetically correlated, and if it is, differences in body size will be slow to evolve (Lande, 1980). Nonetheless, those differences will evolve. To see why, we have to take a detour into quantitative genetic theory.

To keep the theory relatively simple, consider the evolution of several traits that are not sexually dimorphic. We can describe the evolution of these traits in response to selection with the breeder's equation

$$
\mathbf{R}=\mathbf{G} \mathbf{P}^{-1} \mathbf{s}
$$

where $\mathbf{R}$ is the vector of changes in mean trait values from one generation to the next, $\mathbf{G}$ is the genetic variancecovariance matrix (which is where the genetic correlations enter), $\mathbf{P}$ is the phenotypic variance-covariance matrix (and $\mathbf{P}^{-1}$ is its inverse), and $\mathbf{s}$ is the vector of changes in mean trait values before and after selection (compare Lande, 1979). If two or more characters have a genetic correlation of $1, \mathbf{P}$ cannot be inverted and the breeder's equation cannot be directly applied. Perfectly correlated traits must be treated as a single trait.

For all other traits, evolution will continue until $\mathbf{P}^{-1} \mathbf{s}=\mathbf{0}$, unless the process of selection eliminates all genetic variation (making $\mathbf{G}=0$ ). When $\mathbf{P}^{-1} \mathbf{S}=\mathbf{0}$, the mean phenotype in the population is at its selective optimum (Lande, 1979). Notice that $\mathbf{P}^{-1} \mathbf{s}$ is a set of regression coefficients describing the relationship between phenotype and fitness. Genetic correlations play no role in determining the location of the optimum. They affect only the trajectory and the speed by which the optimum is approached.

That's the theory. What about the data? Where's the beef?

Parker and Garant (2005) found that genetic correlations between male and female red jungle fowl were very high; $>0.8$ for every age at which they could be estimated for both tarsus length and body mass. Most correlations were $>0.95$, and some were even estimated to be $>1$ (though large S.E. suggest that the true values are at least slightly $<1$ ). Parker and Garant used the same group of animals to show that, in spite of these very high genetic correlations, males and females differ markedly in growth rates, length of growth period, final body mass, and tarsus length. Theory tells us that high genetic correlations between the sexes will influence the rate and trajectory of body size evolution in red jungle fowl, and these data show us that substantial divergence between the sexes is possible even in traits with very high genetic correlations between the sexes.

There are several lessons for us in these results. First, to understand how gene interactions constrain evolutionary outcomes, we must develop an alternative to the breeder's equation for describing phenotypic evolution (see, for example, Turelli and Barton, 1994). Second, we must find statistics other than genetic correlation to characterize and to measure the strength of genetic constraints. And finally, as we seek to understand the origins and consequences of phenotypic differences, we must remember that large phenotypic differences can arise even when the underlying genotypic differences are small.

KE Holsinger is at the Department of Ecology $\mathcal{E}$ Evolutionary Biology, U-3043, University of Connecticut, Storrs, CT 06269-3043, USA.

\section{E-mail: kent@darwin.eeb.uconn.edu}

Bateman AJ (1948). Heredity 2: 349-368

Lande R (1979). Evolution 33: 402-416.

Lande R (1980). Evolution 34: 292-305.

Parker TH, Garant D (2005). Heredity.

Parker TH, Knapp R, Rosenfeld JA (2002). Animal Behav 64: 291-298.

Turelli M, Barton NH (1994). Genetics 138: 913-941. 